\title{
Translating polar cues
}

During development of the intestinal epithelium, induction of apical-basal cell polarity precedes the establishment of the microvilli-covered apical brush border, which is important for nutrient uptake. Here, Gloerich et al. show that activation of the Ser/Thr kinase LKB1, which promotes cell polarization, triggers a cascade that activates the small $\mathrm{G}$ protein RAP2A and so directly links apical polarity cues and cytoskeletal control of brush border formation.

Expression of LKB1 in single cells is sufficient to trigger brush border formation independently of cell adhesion, and this requires the kinase MST4 and the actin-linking protein ezrin. But how LKB1-mediated polarization is linked to these downstream cytoskeletal events was not known. RAP proteins are best characterized for their role in adhesion, but previously established links between a RAP orthologue and bud formation in yeast led the authors to ask whether RAP might be a good candidate for mediating microvillus formation. Indeed, they found that inactivation of RAP proteins in intestinal epithelial cells inhibited the formation of the brush border in response to LKB1 induction and, by using short hairpin RNAs (shRNAs) to deplete individual RAP proteins, they showed that this was mediated specifically by RAP2A.

The role of RAP2A was specific to events downstream of polarization, as acquisition of apical-basal cell polarity was intact when RAP2A was depleted. RAP2A.GTP levels increased following stimulation of LKB1, and live imaging of cells expressing a reporter for active RAP revealed that it is the RAP2A pool at the apical membrane that is activated.

How does LKB1 promote RAP2A induction? The authors performed an shRNA screen to identify which guanine nucleotide exchange factor (GEF) triggers RAP2A activation, and saw that combined depletion of PDZ domain-containing GEF1 (PDZGEF1) and PDZGEF2 inhibited brush border formation similarly to

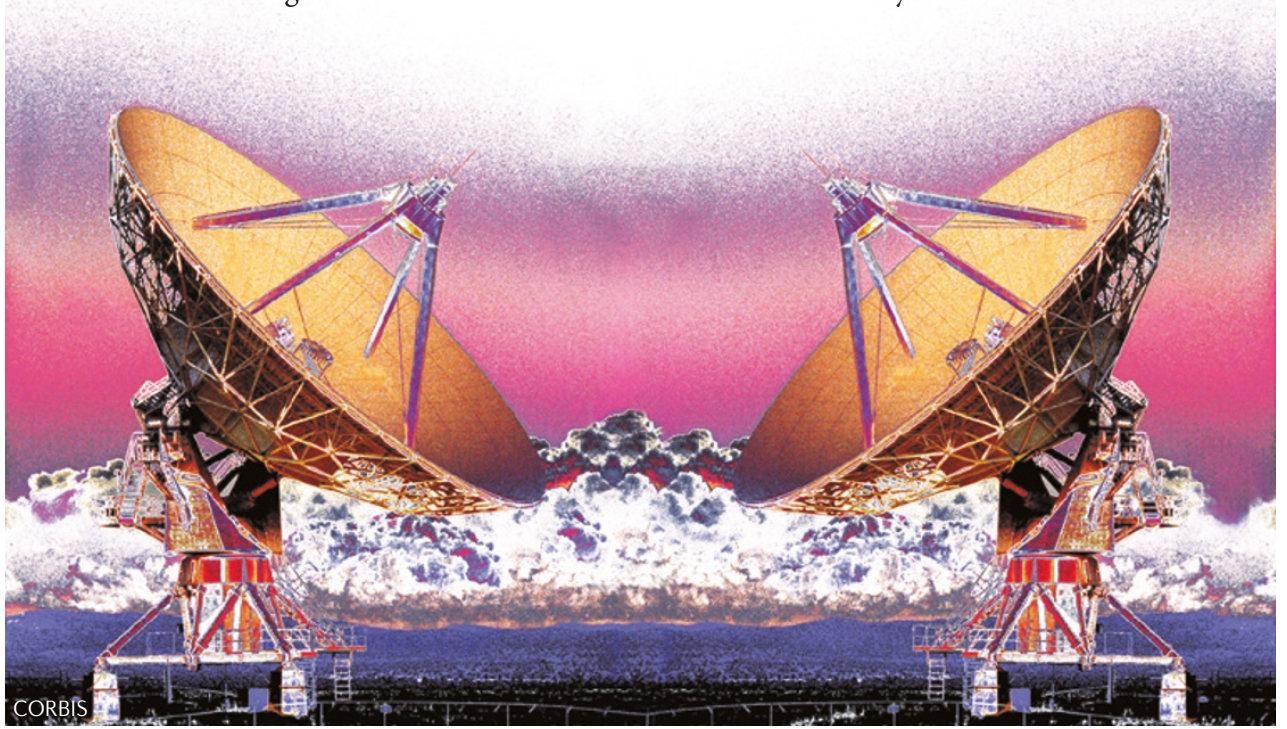

the effects observed when RAP2A was inhibited. These two GEFs were also required for normal activation of RAP2A downstream of LKB1, and YFP fusion proteins of both GEFs redistributed to the apical membrane at sites of RAP2A activation. Next, the authors identified the apical cue that recruits these PDZGEFs. Phosphatidylinositol-4,5bisphosphate $\left(\operatorname{PtdIns}(4,5) \mathrm{P}_{2}\right)$ is known to concentrate at the apical membrane upon cell polarization, and they showed that this phosphoinositide promotes PDZGEF1 and PDZGEF2 relocalization indirectly by associating with phospholipase $\mathrm{D}$ (PLD). PLD generates phosphatidic acid, which they found bound to PDZGEF1 and PDZGEF2. Consistent with this, inhibition of PLD prevented brush border formation.

Last, the authors asked which effectors of RAP2A are required for formation of the brush border. By using shRNA-mediated depletion, they showed that the kinase TNIK is essential for RAP2A-mediated brush border formation, and that it associates with the kinase MST4, which is known to phosphorylate ezrin. TNIK phosphorylated MST4 in vitro and was needed for the proper localization of MST4 in polarized cells. Moreover, a phosphomimetic mutant of ezrin was sufficient to rescue the effects of RAP2A or TNIK loss on brush border formation.

Thus, RAP2A can act independently of adhesion to link LKB1mediated apical-basal cell polarization to brush border formation through kinase-mediated activation of the actin regulator ezrin. This tight coupling of an apical polarity cue with microvillus formation ensures rapid acquisition of cell function at the right time and place.

\section{Alison Schuldt}

ORIGINAL RESEARCH PAPER Gloerich, M. et al. Rap2A links intestinal cell polarity to brush border formation. Nature Cell Biol. 15 Jul 2012 (doi:10.1038/ncb2537) 